\title{
Dissociable Roles for the Hippocampus and the Amygdala in Human Cued versus Context Fear Conditioning
}

\author{
Andreas Marschner, ${ }^{1}$ Raffael Kalisch, ${ }^{1}$ Bram Vervliet, ${ }^{3}$ Debora Vansteenwegen, ${ }^{2}$ and Christian Büchel ${ }^{1}$ \\ ${ }^{1}$ NeuroImage Nord, Department of Systems Neuroscience, University Medical Center Hamburg-Eppendorf, D-20246 Hamburg, Germany, ${ }^{2}$ Department of \\ Psychology, Katholieke Universiteit Leuven, B-3000 Leuven, Belgium, and '3epartment of Psychology, University of Amsterdam, 1012 WX Amsterdam, \\ The Netherlands
}

Lesion studies in animals have identified a critical role of the hippocampus in context fear conditioning. To extend these findings to human volunteers, we used functional magnetic resonance imaging to investigate neural responses associated with context fear conditioning in humans. Our novel conditioning paradigm consisted of aversive electrical shocks (unconditioned stimulus) that were delivered either cue or context related. Differential evoked responses, related to the conditioned stimulus (CS), were found in the anterior cingulate cortex and the bilateral insular cortices, regions that have been implicated in anticipatory anxiety. In case of context conditioning, a similar pattern was observed during the presentation of the entire context. In line with previous conditioning studies, differential responses in the amygdala showed a time by stimulus interaction, suggesting rapid adaptation of CS-specific responses. More importantly, a similar differential decay of activation was observed during context conditioning in the hippocampus, in agreement with a role of the hippocampus in the acquisition phase of human context fear conditioning.

Key words: context; conditioning; hippocampus; amygdala; fMRI; fear

\section{Introduction}

In cued fear conditioning, a neutral conditioned stimulus (CS) is paired with an aversive unconditioned stimulus (UCS) that evokes a strong emotional response. After training, a conditioned response $(\mathrm{CR})$ is elicited by the originally neutral CS. In contrast, context conditioning occurs when aversive events are delivered unsignaled by a discrete CS. In such situations, the context cues are the best predictors for the UCS (Rescorla and Wagner, 1972), and the environment in which conditioning takes place becomes aversive and evokes a fear response (Fanselow, 1980; Grillon et al., 2006; Vansteenwegen et al., 2008).

Considerable evidence indicates that brain structures underlying fear conditioning to an explicit CS ("cued" fear conditioning) and to the context only partially overlap. In rodents, amygdala damage severely impairs both cued and context fear conditioning (Phillips and LeDoux, 1992; LeDoux, 1995), whereas lesions of the hippocampus impair context conditioning but have little effect on cued conditioning (Kim and Fanselow, 1992; Maren et al., 1997; Anagnostaras et al., 1999). The fact that hippocampus-lesioned animals can still produce a CR (in cued paradigms) suggests that the hippocampus is more involved in the acquisition of the context-UCS association (Otto and Poon,

\footnotetext{
Received Jan. 22, 2008; accepted July 17, 2008.

This work was supported by Deutsche Forschungsgemeinschaft (DFG) Grants GRK 1247 (A.M.) and Emmy Noether KA 1623/3-1 (R.K.); C.B. was supported by the Federal Ministry of Education and Research, VolkswagenStiftung, and DFG. We thank Eszter Schoell and Falk Eippert for their helpful comments.

Correspondence should be addressed to Andreas Marschner, Neurolmage Nord, Department of Systems Neuroscience, University Medical Center Hamburg-Eppendorf, Martinistrasse 52, D-20246 Hamburg, Germany. E-mail: a.marschner@uke.uni-hamburg.de.

D0I:10.1523/JNEUROSCl.1651-08.2008

Copyright $\odot 2008$ Society for Neuroscience $\quad 0270-6474 / 08 / 289030-07 \$ 15.00 / 0$
}

2006) than in response expression. In humans, functional neuroimaging studies have shown that cued conditioning involves the amygdala (Büchel et al., 1998; LaBar et al., 1998; Critchley et al., 2002), whereas a recent positron emission tomographic (PET) study has shown that context fear conditioning additionally recruits the hippocampus (Hasler et al., 2007). However, the low temporal resolution of PET prevented a more detailed analysis of the temporal aspects of hippocampal involvement.

In many human cued conditioning studies, amygdala responses decrease rapidly over time (Quirk et al., 1997; Büchel et al., 1998; LaBar et al., 1998), suggesting changes in the CS-UCS association that occur in the early phase of conditioning (i.e., during learning). A similar temporal activation pattern is found in the hippocampus during trace conditioning (Büchel et al., 1999) which, like context conditioning, critically depends on the hippocampus (Eichenbaum, 2004).

In contrast, the anterior insula and the anterior cingulate cortex (ACC) show cued conditioning-related activity that varies little over trials (Büchel et al., 1998; Critchley et al., 2002; Knight et al., 2004), suggesting involvement in CR expression rather than in acquisition.

To test these predictions, we used functional magnetic resonance imaging (fMRI) to identify brain regions associated with context conditioning. The used paradigm installed both context and cued conditioning intermixed in a within-subject design which allowed us to assess the neural correlates of these two types of learning with a high temporal resolution. We hypothesized sustained activity over trials in the insula and the ACC but only transient activity in the amygdala for cue conditioning and the hippocampus for context conditioning. 
A

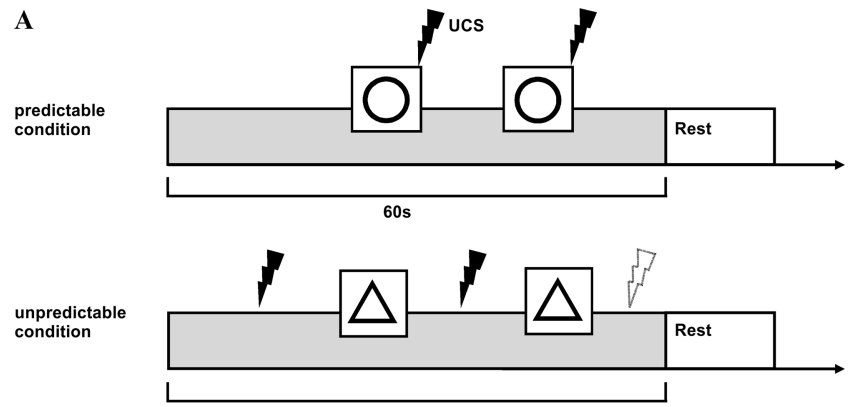

$60 \mathrm{~s}$

B
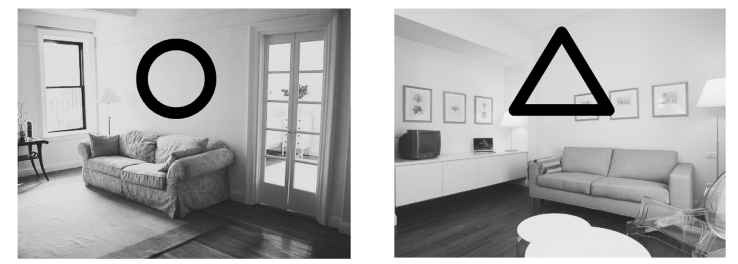

Figure 1. Experimental design. $A$, In both experimental conditions, a background picture of a room served as the context in which learning took place. A geometric figure was repeatedly superimposed on the background picture and served as the discrete CS. In the cue-related condition (top), the discrete CS ("discrete CS+") was always paired with the UCS (electric shock). The UCS was never presented in absence of the discrete CS. In the context-related

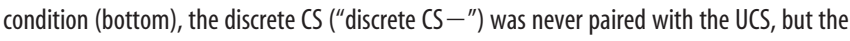
UCS was always presented either before, between, or after the discrete CS $(1,2$, or 3 UCS; mean, 2). In this way, the context ("context $(S+$ ") became a valid predictor of the UCS. To identify correlates of cued conditioning, the discrete CS + was compared with the discrete CS-. To identify correlates of context conditioning, the context $\mathrm{CS}+$ was compared with the context in the cue-related condition ("context CS-"). Throughout each trial, participants continuously rated their current shock expectancy. During the rest periods, they rated the unpleasantness of the UCS that they had received during the preceding trial. $\boldsymbol{B}$, Examples of the two background pictures (context CS) and geometric figures (discrete CS) used. Across participants, rooms and figures were randomly assigned to either the cue-related or the context-related condition.

\section{Materials and Methods}

Participants. Nineteen healthy male participants [mean age $29.5 \pm 9.2$ (SD) years, age range 21-40 years] were enrolled in the study. We restricted our sample to male participants to reduce variability attributable to gender effects in conditioning (Milad et al., 2006). State and trait anxiety were assessed before scanning using the State-Trait Anxiety Inventory (Spielberger et al., 1970; Laux et al., 1981). State anxiety scores ranged from 22 to 37 (mean $29.7 \pm 3.8$ ), trait anxiety scores ranged from 24 to 44 (mean $31.9 \pm 6.1$ ). These values did not deviate from a German normal population (Laux et al., 1981). The study was approved by the Ethics Committee of the Medical Board in Hamburg, Germany, and all subjects gave written informed consent.

Experimental design. The UCS was an electric stimulus consisting of a train of $101 \mathrm{~ms}$ pulses at a frequency of $10 \mathrm{~Hz}$ that were delivered through an electrode pair on the right dorsal forearm. UCS intensity was individually adapted before the experiment to be aversive but not painful (intensity range $4-12 \mathrm{~mA}$ ).

Figure 1 shows the experimental design. Two background pictures of similar but easily distinguishable rooms were used as experimental contexts (context CS + , context CS - ). Two geometric figures (a triangle, a circle) served as discrete CS (discrete CS+, discrete CS-). A block trial lasted $60 \mathrm{~s}$ during which the corresponding background picture (context CS) was continuously present on the screen. The corresponding geometric figure (discrete CS) was presented twice for $5.5 \mathrm{~s}$ each in two time windows (16-18 and 39-41 s). In the cue-related condition, the discrete CS (discrete CS + ) always coterminated with a UCS. This was expected to engender conditioning to the discrete $\mathrm{CS}+$. In the context-related condition, the discrete CS (discrete CS-) was never paired with a UCS. Instead, one, two, or three UCS (mean two) were randomly administered in the time periods before, between, or after the discrete CS. This was expected to engender conditioning to the context CS + . In each partici- pant, each room and each figure were randomly assigned to occur in only one of the two conditions to allow for the formation of clear CS-UCS and context-UCS contingencies. Participants were not informed about these contingencies. Each trial was followed by a $6-8 \mathrm{~s}$ rest period. The entire experiment lasted $33 \mathrm{~min}$.

Before the experiment, participants were familiarized with the stimulus material outside of the scanner. For this purpose, two cue-related and two context-related trials were presented on a computer screen without UCS delivery. During these familiarization trials, participants were also trained to perform both UCS expectancy and UCS unpleasantness ratings (see below).

Subjective ratings. To assess learning, participants rated the likelihood of UCS delivery ("UCS expectancy") at each moment throughout a trial on a discrete scale that was continuously present on the screen. The scale depicted three symbols $(-, 0,+)$, arranged horizontally, that indicated "no shock," "maybe," and "sure shock," respectively. Participants learned the meaning of the symbols before the experiment. The order of the symbols was changed randomly between trials to focus participants' attention and to exclude potential response biases. Ratings were given via three buttons on a response box. The current rating was updated at each button press. To assess a potential effect of conditioning mode on UCS perception, participants rated the perceived unpleasantness of the UCS stimuli that had been delivered in a trial during the subsequent rest period, using the button box and a visual analog scale on the screen (ranging from "no sensation" to "maximum bearable unpleasantness" in 50 steps).

Skin conductance. Skin conductance was measured continuously throughout the experiment via $\mathrm{Ag} / \mathrm{AgCl}$ electrodes placed on the palm of the left hand. The signal was amplified using a CED 2502 amplifier and sampled at $10 \mathrm{~Hz}$ using a CED 1401 analog-digital converter (Cambridge Electronic Design).

Imaging. fMRI was performed on a 3 T MR Scanner (Trio; Siemens) with a standard head coil. Twenty-nine continuous axial slices $(2 \mathrm{~mm}$ thick) were acquired using a $\mathrm{T} 2{ }^{*}$-sensitive gradient echo-planar imaging (EPI) sequence (repetition time, $2.25 \mathrm{~s}$; echo time, $30 \mathrm{~ms}$; slice tilt, $30^{\circ}$; matrix, $104 \times 104$; field of view, $208 \times 208 \mathrm{~mm}$ ). Participants viewed the back-projected stimuli via a $45^{\circ}$ mirror placed atop the head coil. Task presentation and recording of behavioral responses were performed with Cogent2000 (developed at the Wellcome Department of Imaging Neuroscience and Institute of Cognitive Neuroscience, London, UK). Highresolution T1-weighted structural images were also acquired.

Behavioral data analysis. UCS expectancy rating time courses were resampled to $5 \mathrm{~Hz}$ and recoded to values -1 (no shock), 0 (maybe), and +1 (sure shock). Values can vary between -1 and 1 attributable to interpolation and averaging. Effects of cued conditioning were quantified by subtracting average expectancy ratings during discrete CS - presentations from average ratings during discrete CS+ presentations (discrete $\mathrm{CS}+>$ discrete $\mathrm{CS}-$ ). Effects of context conditioning were quantified by subtracting average expectancy ratings during the periods before, between, and after discrete CS presentations in the contextrelated condition from those in the cue-related condition, reflecting the contrast context CS $+>$ context CS - . Effects of background stimuli were quantified by subtracting average expectancy ratings during the first seconds of each trial (before UCS onset) in the cue-related condition from those in the context-related condition. Time courses of contingency learning were assessed by subtracting the average UCS expectancy in the second before discrete CS onset from the maximum rating in the $5 \mathrm{~s}$ after the CS onset resulting in an amplitude value. Mean amplitudes for the first 10 discrete CS were subtracted from the average of the last 10 CS presentations.

Skin conductance data from one subject was lost resulting from technical problems. Time courses were linearly detrended and temporally smoothed [Gaussian kernel; full-width at half-maximal (FWHM), 200 $\mathrm{ms}$ ] to remove scanning artifacts. Similar to the shock expectancy ratings, CS-evoked skin conductance responses (SCR) were quantified by subtracting the average skin conductance in the second before CS onset from the maximum skin conductance in $5 \mathrm{~s}$ after CS onset (Büchel et al., 1998). Data were $\mathrm{z}$ transformed to account for interindividual differences in physiological reactivity. 
Imaging data analysis. Image processing and statistical analyses were performed using SPM5 (statistical parametric mapping 5) (www.fil. ion.ucl.ac.uk/spm). The first five volumes of each time series were discarded to account for T1 equilibrium effects. Volumes were realigned to the first remaining volume to account for movement artifacts, spatially normalized to a standard EPI template, resampled to a voxel size of $3 \times$ $3 \times 3 \mathrm{~mm}$, and finally smoothed using an isotropic Gaussian kernel (FWHM, $8 \mathrm{~mm}$ ).

Statistical analysis was performed separately for each voxel using a general linear model. Context CS + and CS - were modeled as two separate box car regressors (duration, $60 \mathrm{~s}$ ). Discrete CS+ and CS - were separately modeled as events. These four main effect regressors (context

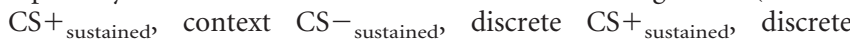
$\mathrm{CS}-{ }_{\text {sustained }}$ ) were intended to capture CS-evoked responses that vary little over trials, as expected for the anterior insula and the ACC/medial prefrontal cortex (mPFC). In addition, we defined four explanatory variables that represented interactions of the main effect regressors with time. These were created by multiplying each main effect regressor with a linear decaying function (context CS $+_{\text {transient }}$, context CS- transient , discrete $\mathrm{CS}+_{\text {transient }}$, discrete $\left.\mathrm{CS}-{ }_{\text {transient }}\right)$, thus modeling CS-evoked responses that decrease over trials, as expected for medial temporal lobe areas. Button presses were modeled as events. All regressors were convolved with a canonical hemodynamic response function.

To identify voxels associated with context conditioning in a temporally sustained manner, a differential contrast comparing the regression parameter estimates for the sustained context CS + and CS - regressors was estimated (contrast context $\mathrm{CS}+_{\text {sustained }}>$ context $\mathrm{CS}-_{\text {sustained }}$ ). To identify voxels associated with cued conditioning in the same way, the corresponding contrast discrete $\mathrm{CS}+_{\text {sustained }}>$ discrete $\mathrm{CS}-_{\text {sustained }}$ was estimated. Analogous contrasts were computed for the interaction regressors. Here, the contrast $\mathrm{CS}+_{\text {transient }}>\mathrm{CS}-_{\text {transient }}$ signifies a steeper decline in activity over CS + compared with CS - trials.

Furthermore, we performed an analysis including additional regressors for the UCS and CS. Finally, we constructed a model in that each block was split into three time periods (before, between, and after the two cues), resulting in three separate regressors. Shock events were orthogonalized against context-conditioning blocks in the last two analyses.

Contrast estimates were tested for group-level significance using voxelwise one-sample $t$ tests. Results were considered significant at $p<0.05$, corrected for multiple comparisons using a family-wise error rate approach. For regions for which we had an a priori hypothesis, correction was limited to a sphere centered around coordinates reported in previous studies ["small volume correction" (SVC)] [left amygdala: $x=-24, y=$ $4, z=-28$; right amygdala: $x=27, y=-2, z=-29$; left anterior insula: $x=-33, y=28, z=-13$; right anterior insula: $x=52, y=26, z=-9$; left ACC/mPFC: $x=-3, y=14, z=37$; right ACC/mPFC: $x=6, y=29$, $z=47$, all from Büchel et al.(1998), transformed into MNI coordinates; left hippocampus: $x= \pm 36, y=-28, z=-7$, from Hasler et al. (2007)]. Sphere radii were $10 \mathrm{~mm}$ for the cortical (anterior insula, ACC/mPFC) and $6 \mathrm{~mm}$ for the subcortical areas (amygdala, hippocampus). For all other brain regions, correction was for all voxels. For illustration purposes, statistical maps are thresholded at $p<0.001$, uncorrected and overlaid on the mean structural image from the group. All activations are reported using $x, y, z$ coordinates in MNI standard space.

\section{Results}

Behavioral data indicated successful cued and context conditioning. Figure 2 shows group-averaged time courses of UCS expectancy ratings during the discrete $\mathrm{CS}+$ (Fig. $2 A$ ), during discrete CS- (Fig. 2B), and for the two context CSs [first seconds of each trial, during which only the background pictures were present (Fig. 3C)]. In the cue-related condition, UCS expectancy increased during presentations of the discrete $\mathrm{CS}+$, which reliably signaled UCS delivery, and quickly decreased afterward. In the context-related condition, UCS expectancy was small during presentations of the discrete $\mathrm{CS}-$ (which had no predictive value) and increased afterward, reflecting the predictive value of the context CS + for UCS delivery. Average expectancy values were
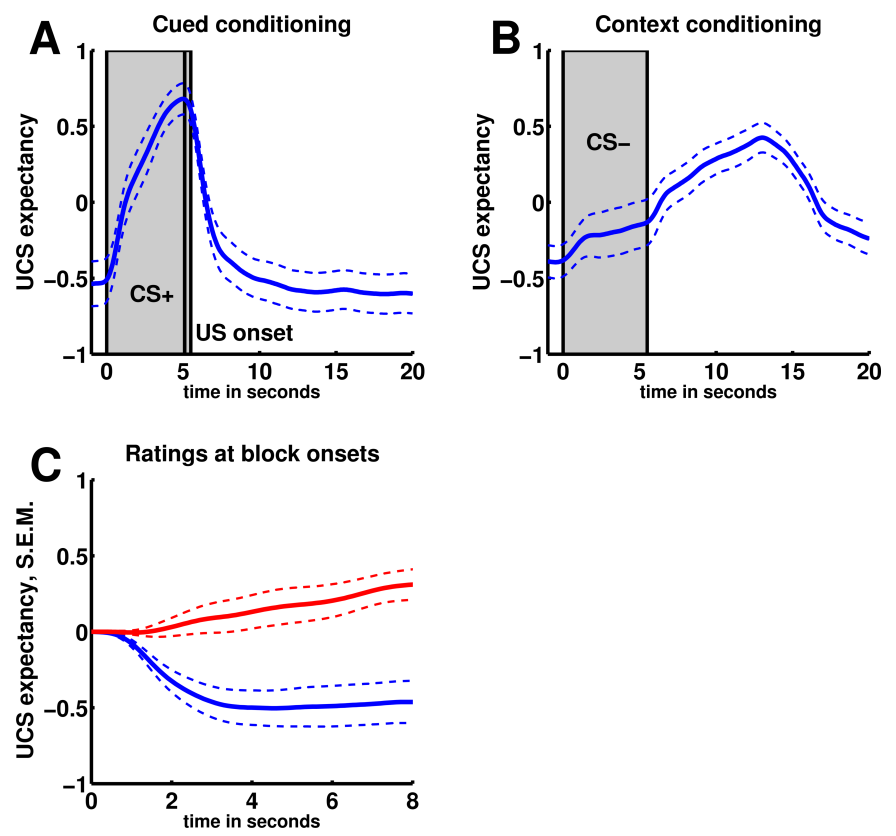

Figure 2. UCS expectancy. Group-averaged UCS expectancy ratings ( \pm SEM) during discrete CS presentations and the subsequent time period during which only the background picture (context CS) was present. Ratings ranged from -1 (no shock), $>0$ (maybe), to +1 (sure shock). $\boldsymbol{A}$, Cue-related condition. $\boldsymbol{B}$, Context-related condition. $\boldsymbol{C}$, Conditioning block onsets (blue, cued conditioning; red, context conditioning).

significantly higher for discrete CS + compared with discrete $\mathrm{CS}-\left(\right.$ contrast discrete $\mathrm{CS}+>$ discrete $\mathrm{CS}-: t_{(18)}=3.7, p=$ 0.0008 ) (Fig. $3 A$ ) and for context CS + compared with context CS - (contrast context CS $+>$ context CS - ; $t_{(18)}=4.6, p=$ 0.00001 ) (Fig. 3A). Likewise, SCRs were significantly higher for discrete CS + compared with discrete CS- (contrast discrete $\mathrm{CS}+>$ discrete CS - ; $t_{(17)}=2.36, p=0.015$ ) (Fig. 3B) and for context CS + compared with context CS - (contrast context CS + $>$ context CS $\left.-; t_{(17)}=1.99, p=0.031\right)($ Fig. $3 B)$.

Shock expectancy ratings during the first seconds of each block before UCS onset were significantly higher for the context $\mathrm{CS}+$ compared with the context $\mathrm{CS}-$, indicating that the participants were able to dissociate the two rooms [mean rating context CS - blocks, $-0.36 \pm 0.38(\mathrm{SD})$, mean rating context $\mathrm{CS}+$ blocks, $\left.0.12 \pm 0.31 ; t_{(18)}=4.0, p=0.0004\right]$. UCS expectancy for the discrete CS+ increased significantly over the course of the experiment reflecting learned associations (mean amplitude for first 10 discrete $\mathrm{CS}+, 0.8 \pm 0.6$; mean amplitude for last 10 discrete CS,$+ 1.3 \pm 0.6 ; t_{(18)}=4.73, p=0.00008$ ) (supplemental Fig. $2 A$, available at www.jneurosci.org as supplemental material). Ratings for the discrete CS - showed a significant decrease over time (mean amplitude for first 10 discrete CS - , $0.5 \pm 0.4$; mean amplitude for last 10 discrete CS,$- 0.3 \pm 0.4 ; t_{(18)}=2.15$, $p=0.023$ ) (supplemental Fig. $2 B$, available at www.jneurosci.org as supplemental material). Although smaller than at the beginning, UCS expectancy still increased monotonically at the end of the experiment as indicated by higher ratings at discrete CSoffset compared with discrete CS- onset (mean baseline rating last 10 discrete $\mathrm{CS}-,-0.26 \pm 0.6$; mean peak rating last 10 discrete CS,$\left.- 0.05 \pm 0.8 ; t_{(18)}=2.05, p=0.028\right)$.

Unpleasantness ratings showed no significant difference between context conditioning and cued conditioning blocks [mean rating cued conditioning, $48.8 \pm 15(\mathrm{SD})$; mean rating context conditioning, $49.2 \pm 15.7 ; t_{(17)}=0.5, p=0.3$ ] (supplemental Fig. 1 , available at www.jneurosci.org as supplemental material). 
A

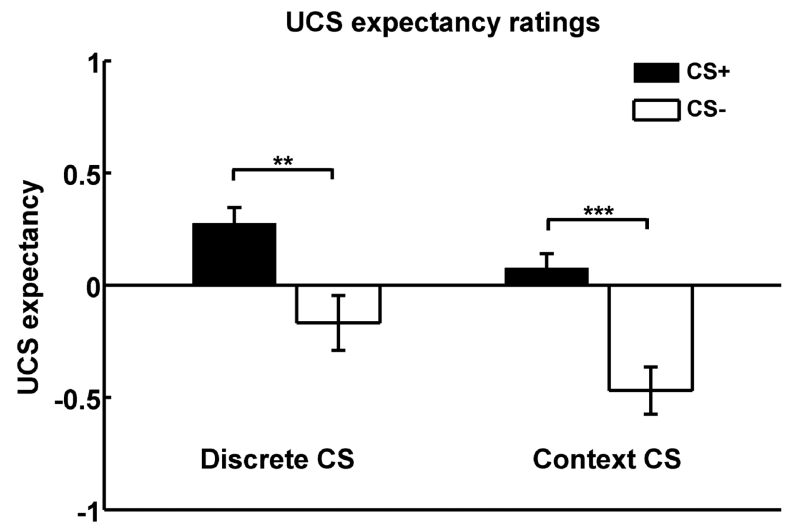

B

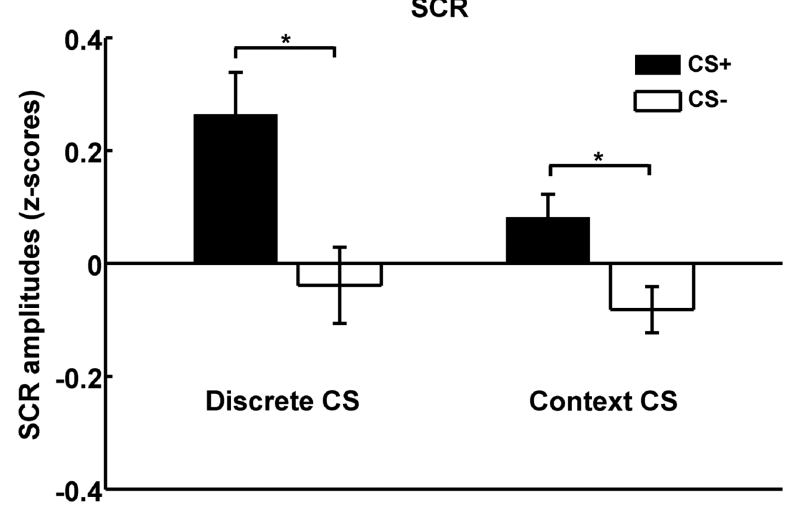

Figure 3. Behavioral and autonomic data showing successful cued and context conditioning. $\boldsymbol{A}, \boldsymbol{B}$, Group-averaged UCS expectancy scores $(\boldsymbol{A})$ and skin conductance responses $(\boldsymbol{B})$ are higher for CS + compared with CS - , indicating successful cued conditioning (left bars, showing responses to discrete $(S)$ and context conditioning (right bars, showing responses to context (S). ${ }^{*} p<0.05 ;{ }^{* *} p<0.01 ;{ }^{* * *} p<0.001$.

Comparing the hemodynamic responses evoked by discrete $\mathrm{CS}+$ and discrete $\mathrm{CS}-\left(\right.$ contrast discrete $\mathrm{CS}+{ }_{\text {sustained }}>$ discrete CS - sustained) showed activation in bilateral anterior insulae (left: $x=-32, y=20, z=-10 ; t_{(18)}=6.46, p=0.001$, corrected for multiple comparisons using SVC; right: $x=44, y=20, z=-8$, $t_{(18)}=7.03, p<0.001 \mathrm{SVC}$ ) and ACC/mPFC (left: $x=-8, y=$ $16, z=38 ; t_{(18)}=9.43, p<0.001$ SVC; right: $x=6, y=14, z=$ $30 ; t_{(18)}=7.92, p=0.018$, corrected for the entire scan volume). Similar sustained activity was observed during context conditioning (contrast context $\mathrm{CS}+_{\text {sustained }}>$ context CS $-_{\text {sustained }}$ ) in bilateral anterior insulae (left: $x=-30, y=24, z=-6 ; t_{(18)}=$ 6.76, $p<0.001$ SVC; right: $x=46, y=26, z=0 ; t_{(18)}=9.41, p<$ 0.001 SVC) and ACC/mPFC (left: $x=-4, y=22, z=44 ; t_{(18)}=$ 4.35, $p=$ SVC; right: $\left.x=6, y=30, z=38, t_{(18)}=7.96, p<0.001\right)$ (Fig. 4, Table 1). This finding supports a role for these areas in CR expression, in line with previous studies (Quirk et al., 1997; Büchel et al., 1998; LaBar et al., 1998).

Activity in the amygdala during cued conditioning showed a decline in activity over discrete CS trials that was more pronounced for the discrete $\mathrm{CS}+$ than for the discrete $\mathrm{CS}-$ (contrast discrete $\mathrm{CS}+_{\text {transient }}>$ discrete $\mathrm{CS}-_{\text {transient }} ; x=22, y=-6, z=$ $-26 ; t_{(18)}=4.11, p=0.010$, corrected for the volume of interest) (Fig. 5A, Table 2). The hippocampus activated in a similarly transient manner during context conditioning (contrast context $\mathrm{CS}+_{\text {transient }}>$ context CS $-_{\text {transient }} ; x=-34, y=-24, z=-14$; $t_{(18)}=4.13, p=0.008$, corrected for the volume of interest) (Fig. $5 B$, Table 2). The two additional analyses modeling CS and UCS
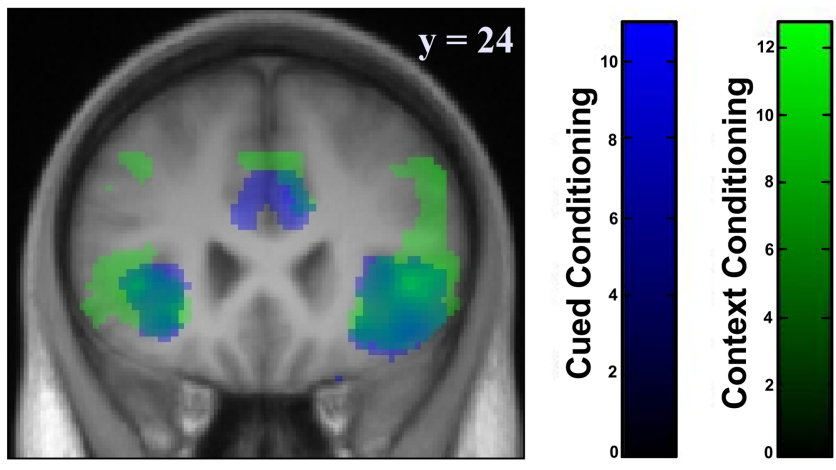

Figure 4. Sustained conditioning-related activity in anterior insula and ACC/mPFC. Sustained activation over $C S$ trials was observed during cued conditioning (contrast discrete $\mathrm{CS}_{\text {sustained }}>$ discrete $\mathrm{CS}-_{\text {sustained }}$, blue) in bilateral anterior and ACC/mPFC. Similarly sustained activity was observed during context conditioning (contrast context $\mathrm{CS}_{\text {sustained }}>$ context CS $-_{\text {sustained, }}$ green). Displayed voxels are thresholded at $p<0.001$, uncorrected. Color bars show $t$ values.
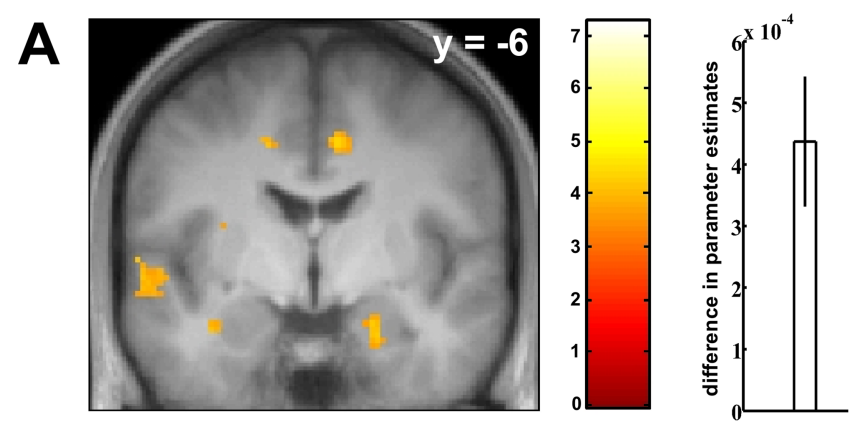

\section{B}
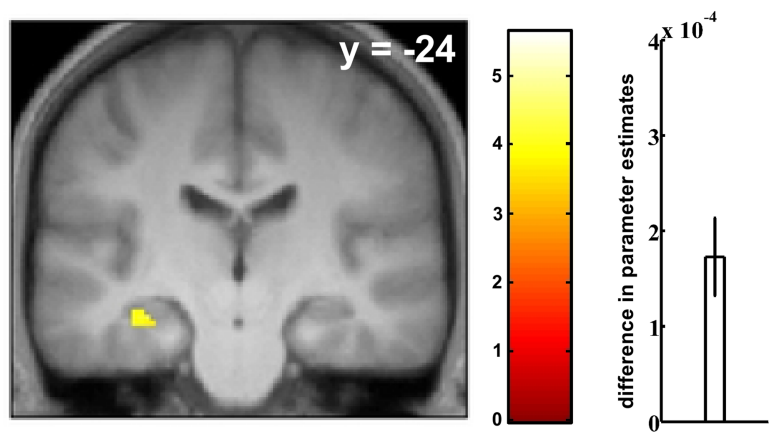

Figure 5. Transient conditioning-related activity in amygdala (cued conditioning) and hippocampus (context conditioning). $\boldsymbol{A}$, Transient activation was observed during cued conditioning (contrast discrete $C S+_{\text {transient }}>$ discrete $C S-_{\text {transient }}$ in right amygdala. $\boldsymbol{B}$, Similarly transient activity was observed during context conditioning in left hippocampus (contrast context $\left(\mathrm{S}+_{\text {transient }}>\right.$ context $\left(S-{ }_{\text {transient }}\right)$. Displayed voxels thresholded at $p<0.001$, uncorrected. Right panels show difference parameter estimates (SEM) reflecting a steeper decline in activation over trials for CS + compared with CS - trials.

events and splitting each block into three time periods did not significantly change the ensuing activation pattern in the hippocampus. These analyses have been added as supplemental information (supplemental Fig. 3, available at www.jneurosci.org as supplemental material).

\section{Discussion}

The present study provides clear evidence for a role of the human hippocampus in aversive context conditioning. During context conditioning blocks, rapid decreases in the differential response of the left hippocampus were observed. The same time course of 
Table 1. Activations sustained over CS trials during cued (left) and context conditioning (right)

\begin{tabular}{|c|c|c|c|c|c|}
\hline \multicolumn{3}{|c|}{ Discrete $\mathrm{CS}+_{\text {sustained }}>\operatorname{discrete} \mathrm{CS}-_{\text {sustained }}$} & \multicolumn{3}{|c|}{ Context CS $+_{\text {sustained }}>$ context $\mathrm{CS}-_{\text {sustained }}$} \\
\hline Region & MNI coordinates & $t_{(18)}$ & Region & MNI coordinates & $t_{(18)}$ \\
\hline L midcingulum & $-8,16,38$ & $5.6^{*}$ & R insula & $46,26,0$ & $5.59 *$ \\
\hline L Rolandic oper & $-48,-26,18$ & $5.84^{* *}$ & L insula & $-30,24,-6$ & $4.71^{*}$ \\
\hline R pallidum & $14,2,-4$ & $5.71^{* *}$ & L parietal inf & $-52,-54,48$ & $5.2^{* *}$ \\
\hline Linsula & $-32,20,-10$ & $4.59 *$ & R mid-ACC & $6,30,38$ & $5.15^{*}$ \\
\hline R Rolandic oper & $-54,2,2$ & $5.54^{* *}$ & $R$ frontal inf orb & $30,28,-8$ & $5.05^{* *}$ \\
\hline L pallidum & $-10,-2,-4$ & $5.37^{* *}$ & L parietal inf & $-54,-48,38$ & $4.92^{* *}$ \\
\hline R insula & $44,20,-8$ & $4.82^{*}$ & L mid-ACC & $-4,22,44$ & $3.55^{*}$ \\
\hline L Heschl & $-48,-10,10$ & $5.22^{* *}$ & & & \\
\hline L temporal sup & $-40,-10,-12$ & $5.2^{* *}$ & & & \\
\hline R Rolandic oper & $46,-24,24$ & $5.18^{* *}$ & & & \\
\hline R thalamus & $4,-14,0$ & $5.15^{* *}$ & & & \\
\hline R midcingulum & $6,14,30$ & $5.14^{* *}$ & & & \\
\hline L thalamus & $-4,-26,4$ & $5.08^{* *}$ & & & \\
\hline L thalamus & $-4,-20,-2$ & $5^{* *}$ & & & \\
\hline R insula & $42,6,6$ & $4.92^{* *}$ & & & \\
\hline R insula & $30,20,-16$ & $4.9^{* *}$ & & & \\
\hline L thalamus & $-8,-4,2$ & $4.89^{* *}$ & & & \\
\hline
\end{tabular}

oper, Operculum orb; sup, superior; inf, inferior; $R$, right; L, left; orb, orbital.

${ }^{*} p<0.05$, after SVC; ${ }^{* *} p<0.05$, corrected for the entire scan volume.

Table 2. Transient activation over trials for the discrete $\mathrm{CS}+$ (left) and for the context CS+ (right)

\begin{tabular}{|c|c|c|c|c|c|}
\hline \multicolumn{3}{|c|}{ Discrete $C S+_{\text {sustained }}>$ discrete $C S-_{\text {sustained }}$} & \multicolumn{3}{|c|}{ Context CS $+_{\text {sustained }}>$ context $C S-_{\text {sustained }}$} \\
\hline Region & MNI coordinates & $t_{(18)}$ & Region & MNI coordinates & $t_{(18)}$ \\
\hline $\begin{array}{l}\text { R amygdala } \\
\text { R enthorinal cortex }\end{array}$ & $\begin{array}{l}22,-6,-26 \\
28,0,-32\end{array}$ & $\begin{array}{l}4.11^{*} \\
3.98^{*}\end{array}$ & L hippocampus & $-34,-24,-14$ & $4.13^{*}$ \\
\hline
\end{tabular}

Time by condition interaction is shown. $R$, Right; $L$, left.

${ }^{*} p<0.05$, after SVC.

activation was shown in the right amygdala comparing the discrete $\mathrm{CS}+$ and discrete $\mathrm{CS}-$. Activation of the ACC and bilateral insulae observed both during the context CS + and for the discrete CS + possibly reflects anticipation of electric shocks.

A crucial feature of this study is the integration of cue conditioning and context conditioning into a single paradigm (Grillon et al., 2006; Vansteenwegen et al., 2008). Pictures of rooms were used as settings in which aversive events were delivered. In both conditioning blocks, the same number of CS and UCS was presented. The conditioning blocks differed only in the level of associability of aversive events. This enabled us to directly assess both types of conditioning.

Behavioral data indicate that the participants learned the CSUCS and context-UCS contingencies. Shock expectancy ratings were significantly higher when the discrete CS + was present during cued conditioning blocks. In contrast, ratings in the context conditioning blocks were significantly lower when the discrete CS - was present. Ratings at the beginning of each block (i.e., before the first UCS) already show a clear dissociation between the two contexts (i.e., being higher for the context conditioning blocks compared with the block in which individual CS are conditioned). This reflects conditioning to differences in contexts and cannot be related to the unpredictability of shock, as no shock has yet occurred at this point in the block.

Our strong behavioral effects also highlight the fact that context conditioning in humans is possible using a unimodally (i.e., visual scene) defined context compared with the multimodally defined context in rodent studies.

As the CS - is never paired with a shock, there is a possibility that this CS becomes a "safety" cue or conditioned inhibitor. However, comparing the first 10 with the last $10 \mathrm{CS}-$ ratings revealed a slight increase in shock expectancy at later stages rather than a decrease as would have been expected if the CS- acted as a safety signal. In addition, during CS - ratings monotonically increase rather than decrease as expected if this CS signaled safety.

The observed pattern of shock ratings was reflected by skin conductance responses. During the onset (first $8 \mathrm{~s}$ ) of the different conditioning blocks, when participants were only exposed to the context defining living room pictures (context CS+), SCRs were stronger to the context conditioning block compared with the cue conditioning block. Together, the behavioral data show that both the discrete CS + and context CS + were identified as potentially dangerous and SCR changes suggest that they also elicited anticipatory anxiety.

Imaging data confirmed our hypothesis of activity sustained over trials in the anterior insula and the ACC/mPFC during both cued and context conditioning. The commonality of both conditions is that a shock is expected in cue conditioning indicated by the discrete CS + and in context conditioning indicated by the context CS + . The expectancy of an aversive stimulus is linked with anticipatory anxiety, and a similar activation pattern has therefore also been observed in studies on anticipatory anxiety which consistently show ACC and insula activation (Ploghaus et al., 1999; Porro et al., 2002; Wager et al., 2004; Kalisch et al., 2006; Nitschke et al., 2006). Aversive stimulation activates mechanisms to prevent future harm by recognizing signals of impending threat (Pavlov, 1927). It has been suggested that the insular cortex has an interoceptive function, monitoring sensations that are important for the integrity of the body (Carter et al., 1999). One of the implicated functions of the ACC is the regulation of attentional resources and pain perception (Hutchison et al., 1999; Buffington et al., 2005). Thus, the anterior cingulate cortex and the insular cortex together play an important role in representing 
the sensory and autonomic experience associated with the anticipation of aversive stimuli.

Decreases in the amygdala response to the discrete CS + are in accord with previous studies on aversive conditioning (Quirk et al., 1997; Büchel et al., 1998; LaBar et al., 1998). These studies suggest that the amygdala encodes sensory contingencies to rapidly learn CS-UCS associations. After acquisition, other regions take over the learned associations and the amygdala disengages. Integrating past experiences for the detection of the correlative structure between contingent events is a prerequisite for fear conditioning and is formally expressed in theoretical learning models of classical conditioning (Rescorla and Wagner, 1972; Dickinson, 1980). According to these theories, a "prediction error" is defined as the discrepancy between the actual and the predicted outcome of a particular learning trial. In a conditioning experiment, learning occurs when the prediction error is high. The rapid decrease of amygdala activation over time found in our study is in line with this model. In the early phase of the experiment, CS-UCS pairings come as a surprise to the volunteer resulting in a high prediction error. Amygdala activation reflects the acquisition of the encountered contingency. Subsequent unconditioned stimuli have now become predictable by the CS, leading to a smaller prediction error and therewith lower amygdala activity. Further evidence for a time-limited role of the amygdala in fear conditioning comes from studies in rodents. Single cell recordings in the dorsal subnucleus of the lateral amygdala in rats during fear conditioning revealed two anatomically distinct cell populations (Repa et al., 2001). Activity of transiently plastic cells, after initial increase in early trials, rapidly decreased during later phases of conditioning. In contrast, long-term plastic cells increased and maintained their firing rates during conditioning. The short term latencies were found in cells, which had previously shown diminished responses late in training (Quirk et al., 1997).

Crucially, a time by condition interaction analysis comparing context conditioning blocks with cued conditioning blocks revealed activation of the hippocampus that decreased over time. Only a few studies have focused on the role of the hippocampus in human context conditioning. Hasler et al. (2007) reported elevated hippocampal activity for context fear using positron emission tomography. The aversive context consisted of a screen indicating that electric shocks would be administered unpredictably over the next trial. In our experimental procedure, instead of verbal instructions, pictures of rooms were used as settings in which aversive events were delivered. Being unaware of the contingencies, participants had to learn the CS-UCS relationship over the course of the experiment. Hence, the current study investigated the learning rather than the expression of anxiety, which is reflected by the imaging data.

The observed temporal pattern in the hippocampus suggests a time-limited role for this area in associative learning. Studies involving experimental hippocampus damage in rats provide evidence for a temporally limited role of the hippocampus in contextual fear memory (Kim and Fanselow, 1992; Maren et al., 1997; Anagnostaras et al., 1999). However, the latter studies investigated these changes in a time frame of days rather than minutes. Adaptation of hippocampal responses over shorter time periods (i.e., minutes) have been observed in human learning studies (Strange et al., 1999). In this study, we report a hippocampal response to learning relevant (i.e., novel) stimuli and an adaptation of these responses over time.

Novelty in this context means presenting a stimulus in the absence of any particular expectation and can be considered a prediction error. Importantly, it has been shown that such pre- diction errors can be accompanied by hippocampal activation (Ploghaus et al., 2000). In analogy, in our experiment volunteers were initially unaware of the context-UCS contingency, leading to a substantial prediction error. The temporal gradient of hippocampal activation as observed in our context conditioning blocks might therefore reflect the decrease of the prediction error during learning (i.e., context conditioning).

The present data are consistent with the hypothesis that amygdala activation plays a more general role in conditioning and that context conditioning additionally recruits the hippocampus. However, in our data amygdala activation during context conditioning only showed a trend toward significance. One possibility for this result might be the fact that during context conditioning, amygdala activity might fluctuate during the block and might therefore be more variable and difficult to assess compared with time locked (to the CS) activation of the amygdala during cue conditioning.

A possible limitation of the present study is that we did not measure direct fear indices, but rather indices of UCS expectancy. However, evidence from a previous behavioral study supports the idea that levels of anxiety in situations of unpredictability are related to the anticipation of threat. Vansteenwegen et al. (2008) also investigated cued and context fear conditioning measuring both online UCS expectancy and startle reflex. Eye blink startle reflexes (elicited by a sudden noise burst) were significantly stronger during phases of higher UCS expectancy, indicating a relationship between these two indices.

Our results support the view that context conditioning recruits the hippocampus. The activation pattern suggests a preferential role of the amygdala and the hippocampus early in conditioning. Periods of higher shock expectancy were reflected by activity in the insula and the ACC, regions that have been implicated in anticipatory anxiety.

\section{References}

Anagnostaras SG, Maren S, Fanselow MS (1999) Temporally graded retrograde amnesia of contextual fear after hippocampal damage in rats: within-subjects examination. J Neurosci 19:1106-1114.

Büchel C, Morris J, Dolan RJ, Friston KJ (1998) Brain systems mediating aversive conditioning: an event-related fMRI study. Neuron 20:947-957.

Büchel C, Dolan RJ, Armony JL, Friston KJ (1999) Amygdala-hippocampal involvement in human aversive trace conditioning revealed through event-related functional magnetic resonance imaging. J Neurosci 19:10869-10876.

Buffington AL, Hanlon CA, McKeown MJ (2005) Acute and persistent pain modulation of attention-related anterior cingulate fMRI activations. Pain 113:172-184.

Carter CS, Botvinick MM, Cohen JD (1999) The contribution of the anterior cingulate cortex to executive processes in cognition. Rev Neurosci 10:49-57.

Critchley HD, Mathias CJ, Dolan RJ (2002) Fear conditioning in humans: the influence of awareness and autonomic arousal on functional neuroanatomy. Neuron 33:653-663.

Dickinson A (1980) A contemporary animal learning theory. Cambridge, UK: Cambridge UP.

Eichenbaum H (2004) Hippocampus: cognitive processes and neural representations that underlie declarative memory. Neuron 44:109-120.

Fanselow MS (1980) Conditioned and unconditional components of postshock freezing. Pavlov J Biol Sci 15:177-182.

Grillon C, Baas JM, Cornwell B, Johnson L (2006) Context conditioning and behavioral avoidance in a virtual reality environment: effect of predictability. Biol Psychiatry 60:752-759.

Hasler G, Fromm S, Alvarez RP, Luckenbaugh DA, Drevets WC, Grillon C (2007) Cerebral blood flow in immediate and sustained anxiety. J Neurosci 27:6313-6319.

Hutchison WD, Davis KD, Lozano AM, Tasker RR, Dostrovsky JO (1999) 
Pain-related neurons in the human cingulate cortex. Nat Neurosci 2:403-405.

Kalisch R, Wiech K, Critchley HD, Dolan RJ (2006) Levels of appraisal: a medial prefrontal role in high-level appraisal of emotional material. Neuroimage 30:1458-1466.

Kim JJ, Fanselow MS (1992) Modality-specific retrograde amnesia of fear. Science 256:675-677.

Knight DC, Cheng DT, Smith CN, Stein EA, Helmstetter FJ (2004) Neural substrates mediating human delay and trace fear conditioning. J Neurosci 24:218-228.

LaBar KS, Gatenby JC, Gore JC, LeDoux JE, Phelps EA (1998) Human amygdala activation during conditioned fear acquisition and extinction: a mixed-trial fMRI study. Neuron 20:937-945.

Laux L, Glanzmann P, Schaffner P, Spielberger CD (1981) Das State-TraitAngstinventar. Weinheim: Beltz.

LeDoux JE (1995) Emotion: clues from the brain. Annu Rev Psychol 46:209-235.

Maren S, Aharonov G, Fanselow MS (1997) Neurotoxic lesions of the dorsal hippocampus and Pavlovian fear conditioning in rats. Behav Brain Res 88:261-274.

Milad MR, Goldstein JM, Orr SP, Wedig MM, Klibanski A, Pitman RK, Rauch SL (2006) Fear conditioning and extinction: influence of sex and menstrual cycle in healthy humans. Behav Neurosci 120:1196-1203.

Nitschke JB, Sarinopoulos I, Mackiewicz KL, Schaefer HS, Davidson RJ (2006) Functional neuroanatomy of aversion and its anticipation. Neuroimage 29:106-116.

Otto T, Poon P (2006) Dorsal hippocampal contributions to unimodal contextual conditioning. J Neurosci 26:6603-6609.

Pavlov IP (1927) Conditioned reflexes: an investigation of the physiological activity of the cerebral cortex: Oxford: Oxford UP.

Phillips RG, LeDoux JE (1992) Differential contribution of amygdala and hippocampus to cued and contextual fear conditioning. Behav Neurosci 106:274-285.
Ploghaus A, Tracey I, Gati JS, Clare S, Menon RS, Matthews PM, Rawlins JN (1999) Dissociating pain from its anticipation in the human brain. Science 284:1979-1981.

Ploghaus A, Tracey I, Clare S, Gati JS, Rawlins JN, Matthews PM (2000) Learning about pain: the neural substrate of the prediction error for aversive events. Proc Natl Acad Sci U S A 97:9281-9286.

Porro CA, Baraldi P, Pagnoni G, Serafini M, Facchin P, Maieron M, Nichelli P (2002) Does anticipation of pain affect cortical nociceptive systems? J Neurosci 22:3206-3214.

Quirk GJ, Armony JL, LeDoux JE (1997) Fear conditioning enhances different temporal components of tone-evoked spike trains in auditory cortex and lateral amygdala. Neuron 19:613-624.

Repa JC, Muller J, Apergis J, Desrochers TM, Zhou Y, LeDoux JE (2001) Two different lateral amygdala cell populations contribute to the initiation and storage of memory. Nat Neurosci 4:724-731.

Rescorla RA, Wagner AR (1972) A theory of Pavlovian conditioning: variations in the effectiveness of reinforcement and non-reinforcement. In: Classical conditioning II. Current Research and Theory. New York: Appleton Century Crofts.

Spielberger CD, Gorsuch RL, Lushene RE (1970) STAI manual for the StaitTrait-Anxiety Inventory. Palo Alto, CA: Consulting Psychologist.

Strange BA, Fletcher PC, Henson RN, Friston KJ, Dolan RJ (1999) Segregating the functions of human hippocampus. Proc Natl Acad Sci U S A 96:4034-4039.

Vansteenwegen D, Iberico C, Vervliet B, Marescau V, Hermans D (2008) Contextual fear induced by unpredictability in a human fear conditioning preparation is related to the chronic expectation of a threatening US. Biol Psychol 77:39-46.

Wager TD, Rilling JK, Smith EE, Sokolik A, Casey KL, Davidson RJ, Kosslyn SM, Rose RM, Cohen JD (2004) Placebo-induced changes in FMRI in the anticipation and experience of pain. Science 303:11621167. 